\title{
Comprehensive Study of MERN Stack - Architecture, Popularity and Future Scope
}

\author{
Bhavyaa $^{1}$, Suhani Gupta ${ }^{1}$, Ms. Vaishali ${ }^{2}$ \\ ${ }^{1}$ Department of Computer Science and Engineering, Dr. Akhilesh Das Gupta Institute of Technology and \\ Management (affiliated to Guru Gobind Singh Indraprastha University), New Delhi, India \\ ${ }^{2}$ Assistant Professor, Department of Computer Science and Engineering, Dr. Akhilesh Das Gupta Institute of \\ Technology and Management (affiliated to Guru Gobind Singh Indraprastha University), New Delhi, India
}

\section{Article Info}

Volume 7, Issue 6

Page Number: 236-240

Publication Issue :

November-December-2021

\section{Article History}

Accepted : 02 Dec 2021

Published : 10 Dec 2021

\section{ABSTRACT}

The biome of JavaScript is constantly evolving and a new framework or library is launched on a regular basis claiming better features. This study focuses on giving guideline to the reader in the process of choosing the best technology by comparing the runtime performance of the MERN (MongoDB, Express, React.js and Node.js) and MEVN (V stands for Vue.js) stack as well as increasing their workability in the job market. To fulfill the purpose, an experiment was conducted to conclude how swift the said stacks perform in building a single page application. For the experiment, two easy to-do applications are built with MERN and MEVN and loading time, adding time, updating and deleting time of the tasks are measured. Moreover, to be able to reveal the software stack trend among the Swedish-based companies, a survey study was conducted. Out of approximately 70 companies contacted, 12 responded. Due to the low number of response on the survey drawing conclusions from the survey and generalizing, the result was challenging. However, the results gathered show that all the respondents use either Vue.js or React.js or both as their client-side software though they are not always combined with Node.js and MongoDB. Other preferred server-side software that are used in combination with React.js or Vue.js are Java, Go and Django. Some of the main factors that the respondents pointed out that affected their choice of software was the ease of learning, the community behind the software, clients' need and availability of that specific software developer.

Keywords: JavaScript, MERN, MEVN, MEAN, React.js, Vue.js, Node.js, angular.js, MongoDB, Express 


\section{INTRODUCTION}

MERN denotes- MongoDB, Express, React, Node, which clearly means the four technologies that build the stack.

MongoDB: An open source, cross platform, document database which signifies that data can be stored employing collections and documents. It consists of data storage which is in JSON (JavaScript Object Notation) format that ensures swift data reciprocity among client and server.

- Express.js: An adjustable, light, server-side, Node.js web application framework which assists in making a quick, safe and comprehensible application.

- React.js: An open-source, client-side JavaScript framework which leverages eutilization of code on more than one platform, for fast implementation.

- Node.js: The leading cross-platform Javascript server. It is assisted by the Google V8 engine which enables you to implement in the front-end simultaneously using JavaScript.

Express and Node build the central application layer. Express.js is the server side web framework and Node.js becomes the accepted and dynamic JavaScript server platform. In order to build any successful application, it is mandatory to put into service the best technology and MERN stack becomes the anchorage for front-end and back-end development.

\section{POPULARITY OF JAVASCRIPT}

JavaScript is a high-level, powerful, amongst the most accepted and widely used programming languages in the world as most websites and web browsers employ this language. As the language is becoming more and more widely accepted, various tools, libraries, and frameworks are made on it, that find the key to many familiar programming complications with a lot more ease and efficiency. Mostly, the continuance of the frameworks/libraries is not very long as a better and newer version gets introduced every 6 months approximately which is claimed to have a better user interface development. To keep up with these rapid changes, developers have to constantly learn new technologies (known as JavaScript Fatigue) and keep themselves updated. Angular.js, Backbone.js, Amber.js, Vue.js and React.js are some very widely used frameworks and libraries and among these, Vue.js and React.js take the top positions. According to the surveys, an increase of $4.3 \%$ had been seen in usage of Vue.js and React.js in 2019, compared to the previous years. Having to choose one of these frameworks and libraries when creating a single page web application, questions arise such as what aspects a developer should consider when choosing one of these frameworks or libraries. Of all the technologies built on JavaScript over the years, we would highlight the run-time performance comparison between the two most dynamic JavaScript libraries- React.js and Vue.js in combination with MongoDB, Node.js and Express. The abbreviations for these two stacks will be used throughout- MERN (MongoDB, Express, React.js, Node.js) and MEVN (MongoDB, Express, Vue.js, Node.js). Furthermore, we shall see the preferential trend between the software stacks among industry practitioners in Sweden to further understanding of trends and relevance between the various stacks. Below are some statistics that show how JavaScript clearly proves to be popular among all the programming languages. 


\begin{tabular}{|llllll|}
\hline Sep 2020 & Sep 2019 & Change & Programming Language & Ratings & Change \\
\hline 1 & 2 & N & C & $15.95 \%$ & $+0.74 \%$ \\
\hline 2 & 1 & $\checkmark$ & Java & $13.48 \%$ & $-3.18 \%$ \\
\hline 3 & 3 & & Python & $10.47 \%$ & $+0.59 \%$ \\
\hline 4 & 4 & & C++ & $7.11 \%$ & $+1.48 \%$ \\
\hline 5 & 5 & & C\# & $4.58 \%$ & $+1.18 \%$ \\
\hline 6 & 6 & & Visual Basic & $4.12 \%$ & $+0.83 \%$ \\
\hline 7 & 7 & & JavaScript & $2.54 \%$ & $+0.41 \%$ \\
\hline 8 & 9 & $\wedge$ & PHP & $2.49 \%$ & $+0.62 \%$ \\
\hline 9 & 19 & A & R & $2.37 \%$ & $+1.33 \%$ \\
\hline 10 & 8 & $\checkmark$ & SOL & $1.76 \%$ & $-0.19 \%$ \\
\hline
\end{tabular}

Figure 1. TIOBE's Popularity Inde

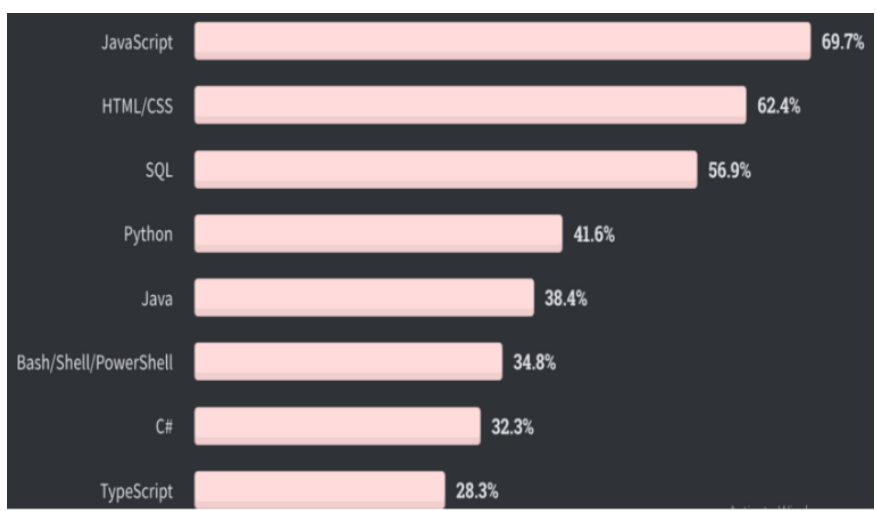

Figure 2. StackOverflow's 2020 Developer's Survey

Now let us see what makes this language so popular?

1) Extraordinary Biome: There are more front-end JavaScript frameworks than what developers usually learn and use. A consistently evolving community is working on the language. There is so much help and support provided to people who work with this language. Although there are many frameworks, which do the same thing, it also helps in making different approaches to solving the same problem and the developer can choose any as per their preference. 2) A bona fide expanding process: As the language follows ECMAScript specifications, it is indirectly controlled by the developers using the language, and is regulated by the TG39 committee. They supervise all the proposals and work on them until they fully develop. All runtimes are updated to the latest version unlike before. III. THE CONCEPT OF MERN STACK
A. How does the MERN stack work The MERN architecture permits an easy build of a 3-layer architecture (Front-end, Back-end and Database) using JavaScript and JSON completely.

- React.js (Front End): It is used for client side applications in HTML. It helps build complicated interfaces using easy components, link them to data at the backend and furnish them as HTML.

- $\quad$ Express.js and Node.js: It has dynamic models for URL routing and handling HTTP requests and responses. Connection to express.js can be made by making XML HTTP requests or GETs or POSTs from react.js front-end.

- MongoDB (Database): For any data-storing app, then an easy to employ database is needed and MongoDB is exactly the best fit. The JSON documents, which are built in react.js, are transferred to express.js where the processing happens and then stored in MongoDB

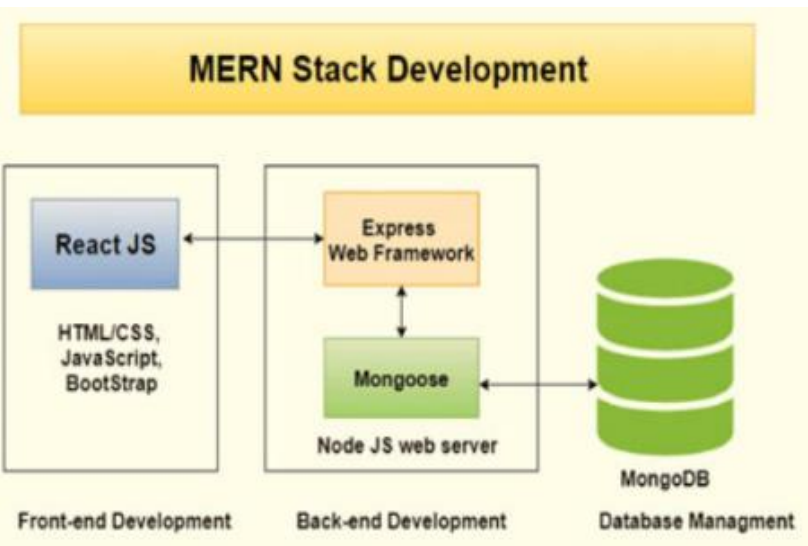

Figure 3. MERN Stack Development

B. Why MERN Stack is becoming so popular The document database at the base of the MERN stack, was immanently made to store JSON data, every aspect of it was made on JSON and Java Script. MongoDB works brilliantly with Node.js and makes storing, operating and displaying application data extremely easy. 
Express.js is a server-side application framework that wraps HTTP requests and responses, and makes it easy to map URLs to server-side functions. React.js is

a frontend JavaScript framework for building interactive user interfaces in HTML, and communicating with a remote server. Like any web stack. Anything can be built in MERN, however, It is best suitable for JSON heavy, cloud native cases that have dynamic web interfaces.
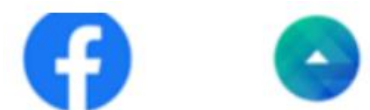

\section{Facebook} Android - ios

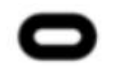

Oculus Android - ios

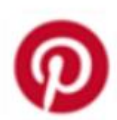

Pinterest Android - ios
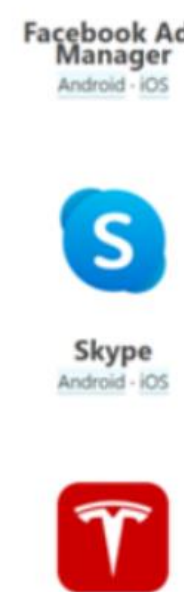

Tesla

Android - ios
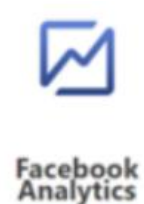

Android - iOS

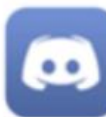

Discord

ios

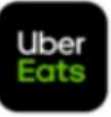

Uber Eats

Nering Uber Eats with
- Low learning curve: MERN stack is easy to learn and operate but if the developer knows JavaScript then it is even easier to learn.

- Pool of Talent available: As MERN is very popular because of its a affiliation with JavaScript; it is easy for startups to find talent for their projects.

- Open Source: All the four tiers of MERN stack are open source which means startups don't face any licensing problems.

- Testing tools: MERN has its own testing tools set so it's convenient for new developers to test the software.
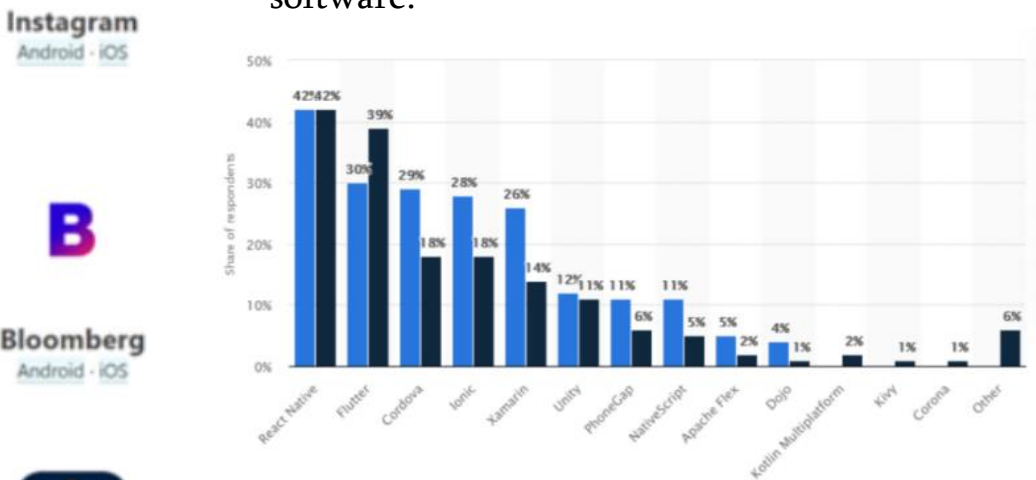

Figure 5

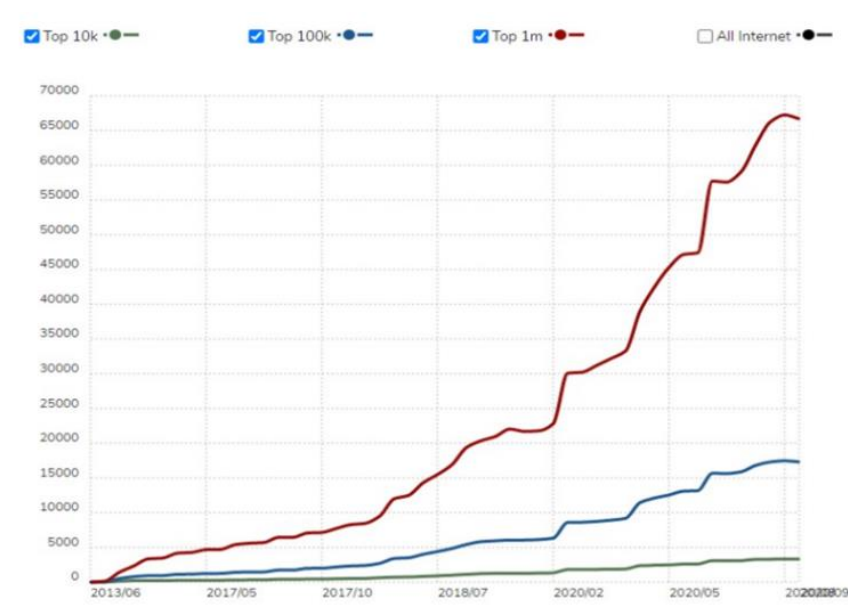

Figure 6. React Usage Statistics

\section{III.CONCLUSION} destination for all technologies and tools.

- $\quad$ MVC Architecture support: MERN assists Model View Controller architecture; this makes developers to develop workflow easily.
This leads us to the end of our research paper where we precisely discussed MERN and then pivoted to its benefits and popularity. For any startup or developer 
Bhavyaa et al Int. J. Sci. Res. Comput. Sci. Eng. Inf. Technol, November-December-2021, 7 (6) : 236-240

to reach the next height, you need a firm set of skills and keep yourself updated with new technologies and tools and MERN stack is definitely on top of the list. The app development company would surely keep all these points and build an incredible platform for apps assisted with this wonderful technology. MERN stack uses high-end tools for building apps, and along with extensive support and built-in testing tool kit, etc, this technology tops the chart in terms of usage and scalability.

\section{REFERENCES}

[1]. https://www.mindinventory.com/blog/mean-st ack-vs-mern-stack/

[2]. https://www.youtube.com/watch?v=5XlYQR $\mathrm{GgYrY}$

[3]. https://ijsrcseit.com/paper/CSEIT217337.pdf

[4]. https://www.datasciencecentral.com/profiles/bl ogs/mern-vs-mean-which-stack-to-use-in-202 1

[5]. https://javascript.plainenglish.io/why-using-th e-mern-stack-is-a-popular-start-up-app-idea-8 7a4a0e8ebbf

[6]. https://www.mongodb.com/mern-stack

[7]. https://javascript.plainenglish.io/why-using-th e-mern-stack-is-a-popular-start-up-app-idea-8 7a4a0e8ebbf

[8]. https://www.ijert.org/research/performance-op timization-using-mern-stack-on-web-applicati on-IJERTV10IS060239.pdf

[9]. https://blog.bitsrc.io/whats-in-the-future-for-ja vascript-b58fc736f1c3

[10]. https://blog.bitsrc.io/whats-in-the-future-for-ja vascript-b58fc736f1c3

[11]. https://www.jetir.org/view?paper=JETIR21058 81

[12]. https://www.jetir.org/view?paper=JETIR21061 72

[13]. http://ijsrd.com/Article.php?manuscript=IJSR DV5I10382
[14]. https://www.ijmtst.com/volume7/issue05/1.IJ MTST0705003.pdf

[15]. https://www.ijrra.net/Vol5issue1/IJRRA-05-0126.pdf

[16]. https://www.geeksforgeeks.org/mern-stack/

[17]. https://www.mongodb.com/languages/mern-st ack-tutorial

[18]. https://blog.logrocket.com/mern-stack-tutorial/

[19]. https://www.mobinius.com/blogs/what-to-cho ose-full-stack-vs-mean-stack-vs-mern-stack-in2021/

[20]. https://hackernoon.com/most-popular-technolo gy-stack-to-choose-from-full-stack-vs-mean-st ack-vs-mern-stack-in-2019-d12c0a17439a

[21]. https://medium.com/@devathon_/mean-vs-me rn-stack-development-5ba3e517bc68

[22]. https://generalassemb.ly/blog/what-makes-jav ascript-so-popular/\#: : :text=There\%20are\%20 over\%201.8\%20Billion,to\%20Github's\%2020 20\%20Octoverse\%20Report

[23]. https://www.codecademy.com/resources/blog/j avascript-history-popularity/

[24]. https://www.linkedin.com/pulse/why-mern-sta ck-our-preferred-choice-saas-startups-surojit-s aha?trk=public_profile_article_view\#: :text= High\%20Scalability\%3A\&text=MERN\%20st ack\%20is\%20highly\%20flexible,and\%20com patible\%20with\%20cloud\%20storage.

\section{Cite this article as :}

Bhavyaa, Suhani Gupta, Ms. Vaishali, "Comprehensive Study of MERN Stack - Architecture, Popularity and Future Scope ", International Journal of Scientific Research in Computer Science, Engineering and Information Technology (IJSRCSEIT), ISSN : 2456-3307, Volume 7 Issue 6, pp. 236-240, November-December 2021. Available at doi : https://doi.org/10.32628/CSEIT217630 Journal URL : https://ijsrcseit.com/CSEIT217630 